\title{
Multimodal evaluation of CSF dynamics following extradural decompression for Chiari malformation Type I
}

\author{
Jennifer L. Quon, BS, Ryan A. Grant, MD, MS, and Michael L. DiLuna, MD \\ Yale University School of Medicine, Department of Neurosurgery, New Haven, Connecticut
}

OBJECT Extradural decompression is a minimally invasive technique for treating Chiari malformation Type I (CM-I) that avoids the complications of dural opening. While there is no agreement on which surgical method is optimal, mounting evidence demonstrates that extradural decompression effectively treats clinical symptoms, with a minimal reoperation rate. Neurological symptoms such as headache may be related to obstructed flow of CSF, and one aspect of successful extradural decompression is improved CSF dynamics. In this series, the authors report on their use of phase-contrast cine flow MRI to assess CSF flow as well as satisfactory decompression.

METHODS The authors describe their first surgical series of 18 patients with CM-I undergoing extradural decompression and correlate clinical improvement with radiological changes. Patients were categorized as having complete, partial, or no resolution of their symptoms. Posterior fossa area, cisterna magna area, and tonsillar herniation were assessed on T2-weighted MRI, whereas improvement of CSF flow was evaluated with phase-contrast cine flow MRI. All patients received standard pre- and postoperative MRI studies; 8 (44.4\%) patients had pre- and postoperative phase-contrast cine, while the rest underwent cine studies only postoperatively.

RESULTS All 18 patients presented with symptomatic CM-I, with imaging studies demonstrating tonsillar herniation $\geq$ $5 \mathrm{~mm}$, and 2 patients had associated syringomelia. All patients underwent suboccipital decompression and C-1 laminectomy with splitting of the dura. Patients with complete resolution of their symptoms had a greater relative increase in cisterna magna area compared with those with only partial improvement $(p=0.022)$. In addition, in those with complete improvement the preoperative cisterna magna area was smaller than in those who had either partial $(0.020)$ or no $(0.025)$ improvement. Ten (91\%) of the 11 patients with improved flow also had improvement in their symptoms. There was 1 postoperative complication of dysphagia and dysphonia. None of the patients have required a second operation.

CONCLUSIONS Extradural decompression has the potential to be the first-line treatment for $\mathrm{CM}$-I but has been lacking an objective measure by which to assess surgical success as well as the need for reoperation. An increase in the CSF spaces and improved CSF dynamics may be associated with resolution of clinical symptoms. Including cine imaging as part of routine pre- and postoperative evaluation can help identify which patients are most likely to benefit from surgery. http://thejns.org/doi/abs/10.3171/2014.10.SPINE1433

KEY WORDS Chiari malformation Type I; extradural decompression; cine flow MRI; cervical

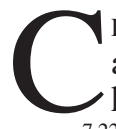

HIARI malformation Type I (CM-I) is an anatomical abnormality characterized by cerebellar tonsillar herniation of $\geq 5 \mathrm{~mm}$ through the foramen magnum, ${ }^{7,22}$ often associated with headache, long tract signs, bulbar symptoms, and other neurological impairment. Symptoms are thought to derive from impaired dynamic CSF flow ${ }^{15,22}$ or direct compression of local anatomy. ${ }^{16,23}$ While surgical techniques to widen the posterior fossa, decompress neural structures, and improve CSF flow have been widely described, ${ }^{16} \mathrm{CM}-\mathrm{I}$ has no single definitive treatment. ${ }^{7,13,15}$ The classic method of decompression includes a suboccipital craniectomy and C-1 laminectomy, with or without autograft or allograft duraplasty. Numerous additional surgical techniques have been described in the literature, ${ }^{2}$ including manipulation or resection of the cerebellar tonsils, as well as stent or shunt treatment of the

ABBREVIATIONS CM-I = Chiari malformation Type I; PFV = posterior fossa volume.

SUBMITTED January 9, 2014. ACCEPTED October 14, 2014.

INCLUDE WHEN CITING Published online March 6, 2015; DOI: 10.3171/2014.10.SPINE1433.

DISCLOSURE The authors report no conflict of interest concerning the materials or methods used in this study or the findings specified in this paper. 
fourth ventricle and syrinx to improve CSF dynamics. ${ }^{20}$ However, these techniques are not without complications, which are often related to penetrating the dura or bleeding. ${ }^{9}$

First described in 1993 by Isu et al., extradural CM-I decompression - that is, the removal of only the outer layer of the dura mater-is an alternative surgical technique that avoids the risks of CSF leak and vascular injury, while allowing decompression of the expandable inner dural layer., ${ }^{7,13}$ In this dura-splitting technique, there must be 2 identifiable dural leaflets, with an extensive outer leaflet resection performed to allow relaxation of the inner dural layer. ${ }^{15}$ In CM-I, symptoms can recur even with adequate surgical intervention of any kind, ${ }^{15,17}$ and therefore the surgeon must ensure sufficient execution of surgical technique to minimize potential treatment failure. Given that a significant portion of the underlying pathology may be secondary to CSF flow obstruction, ${ }^{21}$ cine flow MRI provides an important tool to assess CSF flow. With this, we show that phase-contrast cine flow MRI can contribute to the preoperative evaluation as well as corroborate whether a satisfactory decompression has occurred.

In this report, we describe our first experience of 18 patients with CM-I undergoing extradural decompression, for whom we assessed posterior fossa decompression as well as CSF dynamics using both T2-weighted and phasecontrast cine flow MRI. We believe that cine flow imaging to evaluate CSF flow will become increasingly useful for the multimodal evaluation of a successful extradural decompression.

\section{Methods}

\section{Patient Population}

Eighteen patients with CM-I were treated surgically by the senior author (M.L.D.). Their age at admission ranged from 10 to 50 years (mean 28.8 years). Three (16.7\%) of the 18 patients were male, and 15 (83.3\%) were female (Table 1).

\section{Neurological Assessment}

Symptoms and results of clinical examinations were obtained from a retrospective review of the surgeon's clinic notes. Functional grade was determined using a grading scale previously reported by Noudel et al. ${ }^{19} \mathrm{~Pa}-$ tients received an initial postoperative follow-up visit in the clinic between 5 and 40 days (mean 16.27 days), with continual follow-up at several-month intervals thereafter. Clinical improvement was determined by an author who only assessed clinical symptoms and was blinded to the radiological results. Patients' symptoms were considered to have improved if symptom improvement was clearly documented in any postoperative clinic notes. Patients were categorized as having no, partial, or complete resolution of their symptoms based on their last documented visit.

\section{Radiological Studies}

All radiological measurements and assessment were performed by an author who was blinded to the clinical symptoms and outcome. The diagnosis of CM-I was made using MRI and was defined as tonsillar herniation of at least $5 \mathrm{~mm}$ below the level of the foramen magnum. Posterior fossa and cisterna magna area were measured using T2-weighted imaging at the midsagittal plane. The method for determining posterior fossa area was based on previously reported methods of measuring posterior fossa $^{19}$ and cisterna magna ${ }^{23}$ volumes. Narrowing of CSF spaces anterior and posterior to the spinal cord was assessed on T2-weighted MRI. Postoperative improvement in CSF flow was demonstrated by comparing T2 signal on pre- and postoperative images. Phase-contrast cine flow imaging was compared pre- and postoperatively, when available, and assessed on all patients postoperatively to confirm adequate CSF flow. Postoperative MRI with cine was obtained approximately 3 months after surgery.

\section{Surgical Technique}

All 18 patients underwent extradural surgical CM-I decompression in the prone position. A standard suboccipital craniectomy was performed to ensure a wide decompression of the cerebellar hemispheres, brainstem, and midline structures. In all cases, a C-1 laminectomy was also performed to decompress the cervical spinal cord. The atlanto-occipital ligament was divided, and the underlying outer dura leaflet was incised and reflected radially, while maintaining the integrity of the inner leaflet of the dura. Closure was then performed in a standard multilayer fashion, similar to that used in intradural procedures, to guard against any potential CSF leak not noted intraoperatively.

\section{Statistical Analysis}

A univariate analysis was used to compare groups, and $\mathrm{p}<0.05$ was considered significant.

\section{Results}

\section{Demographic Characteristics, Presenting Ailments, and} Preoperative Radiographic Assessment

As stated earlier, there were 3 males (16.7\%) and 15 females $(83.3 \%)$ in our patient group, with their average age being 28.8 years (range $10-50$ years). All 18 patients had headache as a presenting symptom, with subtypes including Valsalva/exertional, cluster, migraine, and tension; the locations were frontal, temporal, and occipital. Notably, many patients initially had multiple headache types. Additional reported symptoms included upper- and lowerextremity pain and/or numbness, neck pain, dizziness and vertigo, imbalance, visual symptoms, photosensitivity/ phobia, nausea, fatigue, and difficulty breathing as well as compressive symptoms such as dysphagia and hiccups (Table 2). Twelve patients reported exacerbation of their symptoms with coughing, sneezing, exertion, or other Valsalva-like maneuvers. Only 3 patients had Hoffman's and/ or Lhermitte's signs. Other neurological findings included downbeating nystagmus $(\mathrm{n}=4)$, hypophonia $(\mathrm{n}=2)$, and diminished sensation in the extremities $(n=1)$. Four patients had pain with and restriction of neck movement. Patients' functional grades ranged from normal findings on clinical examination with slight impairments (I) to disabled but with the persistence of autonomy (IV).

The posterior fossa area ranged from 2489.57 to 
TABLE 1. Summary of demographic and clinical characteristics in 18 cases

\begin{tabular}{|c|c|c|c|c|c|c|c|c|c|}
\hline \multirow{2}{*}{$\begin{array}{l}\text { Case } \\
\text { No. }\end{array}$} & \multirow{2}{*}{$\begin{array}{c}\text { Age (yrs), } \\
\text { Sex }\end{array}$} & \multirow[b]{2}{*}{ Headache } & \multirow[b]{2}{*}{ Lhermitte's Sign } & \multirow[b]{2}{*}{ Hoffman's Sign } & \multirow[b]{2}{*}{ Syrinx } & \multicolumn{2}{|c|}{ Functional Assessment } & \multirow[b]{2}{*}{ Clinical Improvement } & \multirow[b]{2}{*}{ Postop Complications } \\
\hline & & & & & & Preop & Postop & & \\
\hline 1 & $28, F$ & Y & $\mathrm{N}$ & Left $N$, right $Y$ & $\mathrm{~N}$ & IV & II & $\mathrm{P}+$ & $\mathrm{N}$ \\
\hline 2 & $32, \mathrm{~F}$ & Y & $\mathrm{N}$ & $\mathrm{N}$ & $\mathrm{N}$ & I & I & $\mathrm{N}$ & $\mathrm{N}$ \\
\hline 3 & $21, M$ & Y & $\mathrm{N}$ & $\mathrm{N}$ & $\mathrm{N}$ & II & I & $\mathrm{N}$ & $\mathrm{N}$ \\
\hline 4 & $17, \mathrm{~F}$ & $\mathrm{Y}$ & $\mathrm{N}$ & $\mathrm{N}$ & $\mathrm{N}$ & II & I & $\mathrm{P}++$ & $\mathrm{N}$ \\
\hline 5 & $34, \mathrm{~F}$ & $\mathrm{Y}$ & $\mathrm{N}$ & $\mathrm{N}$ & $\mathrm{N}$ & III & I & $\mathrm{P}++$ & $\mathrm{N}$ \\
\hline 6 & $39, \mathrm{~F}$ & $\mathrm{Y}$ & $\mathrm{N}$ & Y & $\mathrm{N}$ & III & I & $\mathrm{P}++$ & Dysphagia, hypophonia \\
\hline 7 & $42, \mathrm{~F}$ & $\mathrm{Y}$ & $\mathrm{N}$ & $\mathrm{N}$ & $\mathrm{N}$ & 1 & 1 & $\mathrm{P}$ & $\mathrm{N}$ \\
\hline 8 & $50, \mathrm{~F}$ & $Y$ & $\mathrm{~N}$ & $\mathrm{~N}$ & $\mathrm{~N}$ & I & 0 & C & $\mathrm{N}$ \\
\hline 9 & $14, \mathrm{~F}$ & $Y$ & $\mathrm{~N}$ & $\mathrm{~N}$ & $\mathrm{~N}$ & I & 1 & $\mathrm{P}+$ & $\mathrm{N}$ \\
\hline 10 & $44, \mathrm{~F}$ & $Y$ & $Y$ & $Y$ & $\mathrm{~N}$ & IV & 1 & $\mathrm{P}+$ & $\mathrm{N}$ \\
\hline 11 & $29, \mathrm{M}$ & $Y$ & $\mathrm{~N}$ & $\mathrm{~N}$ & $\mathrm{~N}$ & 1 & I & $\mathrm{P}++$ & $\mathrm{N}$ \\
\hline 12 & $10, \mathrm{~F}$ & $Y$ & $\mathrm{~N}$ & $\mathrm{~N}$ & $Y$ & II & I & P++ & $\mathrm{N}$ \\
\hline 13 & $33, F$ & $Y$ & $\mathrm{~N}$ & $\mathrm{~N}$ & $\mathrm{~N}$ & II & 1 & $\mathrm{P}++$ & $\mathrm{N}$ \\
\hline 14 & $30, F$ & $Y$ & $\mathrm{~N}$ & $\mathrm{~N}$ & $\mathrm{~N}$ & II & 1 & $\mathrm{P}+$ & $\mathrm{N}$ \\
\hline 15 & $15, F$ & $Y$ & $\mathrm{~N}$ & $\mathrm{~N}$ & $\mathrm{~N}$ & II & 1 & $P$ & $\mathrm{~N}$ \\
\hline 16 & $19, \mathrm{~F}$ & $Y$ & $\mathrm{~N}$ & $\mathrm{~N}$ & $\mathrm{~N}$ & II & 0 & $C$ & $\mathrm{~N}$ \\
\hline 17 & $42, \mathrm{~F}$ & $Y$ & $\mathrm{~N}$ & $\mathrm{~N}$ & $Y$ & II & I & $\mathrm{P}$ & $\mathrm{N}$ \\
\hline 18 & $20, M$ & $Y$ & $\mathrm{~N}$ & $\mathrm{~N}$ & $\mathrm{~N}$ & II & I & $\mathrm{P}++$ & $\mathrm{N}$ \\
\hline
\end{tabular}

$\mathrm{C}=$ complete improvement; $\mathrm{N}=$ no; $\mathrm{P}=$ partial improvement; $\mathrm{P}+=$ slight improvement; $\mathrm{P}++$ = good improvement; $\mathrm{Y}=$ yes.

$3576.52 \mathrm{~mm}^{2}$ (mean $2997.78 \mathrm{~mm}^{2}$ ). Notably, 10 patients (55.6\%) had no measurable cisterna magna, with areas ranging from 0 to $15.53 \mathrm{~mm}^{2}$ (mean $4.95 \mathrm{~mm}^{2}$ ). Cerebellar tonsillar herniation ranged from 4.5 to $20.99 \mathrm{~mm}$ (mean $9.86 \mathrm{~mm}$ ). Two patients (11.1\%) had syrinxes associated with their condition (Table 1).

\section{Neurological Outcomes}

Two (11.1\%) of the 18 patients reported a complete resolution of their symptoms, $14(77.8 \%)$ reported partial resolution, and 2 reported no improvement in their symp-

TABLE 2. Preoperative symptoms

\begin{tabular}{lc}
\hline \multicolumn{1}{c}{ Symptom } & No. of Patients \\
\hline Headache (all types) & 18 \\
\hline Valsalva exacerbation of symptoms & 12 \\
\hline UE \&/or LE pain, numbness, or paresthesias & 9 \\
\hline Neck pain & 12 \\
\hline Compressive symptoms & \\
\hline$\quad$ Dysphagia & 5 \\
\hline Hiccups & 1 \\
\hline Nausea & 5 \\
\hline Dizziness/vertigo & 10 \\
\hline Imbalance & 4 \\
\hline Photosensitivity/-phobia & 2 \\
\hline Visual symptoms & 2 \\
\hline Fatigue & 1 \\
\hline Difficulty breathing & 1 \\
\hline LE = lower-extremity; UE = upper-extremity. &
\end{tabular}

toms. Two patients had a recurrence of some symptoms that had initially improved. The latency to begin seeing an improvement in symptoms ranged from 7 days to 9 months. The change in functional grade following treatment ranged from no difference to an improvement of 3 grades. Patients' postoperative functional grades ranged from 0 to II.

Sixteen patients $(88.9 \%)$ noted some improvement in their headaches. Six patients had complete resolution of their headaches, 5 had decreased frequency, 2 were able to control their headaches with over the counter analgesics, and 4 (including one of the patients with decreased headache frequency) had resolution of some, but not all, headache subtypes. Refractory headache types included migraine $(\mathrm{n}=2)$, exertional headaches $(\mathrm{n}=1)$, and temporally located headaches $(n=1)$. Other symptoms that were alleviated postoperatively included upper- and lowerextremity sensory changes $(n=7)$, neck pain $(n=4)$, dizziness/vertigo $(\mathrm{n}=5)$, visual symptoms $(\mathrm{n}=1)$, and dysphagia $(\mathrm{n}=2)($ Table 3$)$.

Two patients did not report any improvements in symptoms within 1 year of follow-up. Patients who did not report immediate clinical improvement continued to experience headaches $(n=2)$, neck pain/stiffness $(n=2)$, dizziness $(\mathrm{n}=1)$, and photophobia $(\mathrm{n}=1)$. No patient has had a worsening of symptomatology following surgery.

\section{Radiological Outcomes}

Seventeen patients $(94.4 \%)$ had an increase in posterior fossa area following decompression. The postoperative posterior fossa area ranged from 2746.23 to $3572.89 \mathrm{~mm}^{2}$ (mean $3193.68 \mathrm{~mm}^{2}$ ) with an average increase of 209.31 
TABLE 3. Postoperative improvement in symptoms

\begin{tabular}{lc}
\hline \multicolumn{1}{c}{ Symptom } & No. of Patients \\
\hline Headache (all types) & 16 \\
\hline UE \&/or LE pain, numbness, or paresthesias & 7 \\
\hline Neck pain & 4 \\
\hline Dizziness/vertigo & 5 \\
\hline Visual symptoms & 1 \\
\hline Dysphagia & 2 \\
\hline
\end{tabular}

$\mathrm{mm}^{2}$ compared with preoperative areas (Table 4). All 18 patients had an increase in cisterna magna area following decompression, with an average change of $41.84 \mathrm{~mm}^{2}$. The postoperative cisterna magna area ranged from 10.60 to $119.56 \mathrm{~mm}^{2}$ (mean $33.90 \mathrm{~mm}^{2}$ ). Following decompression, tonsillar herniation ranged from 4 to $18.67 \mathrm{~mm}$ (mean $8.015 \mathrm{~mm}$ ). Sixteen patients had an improvement in tonsillar herniation; in 1 patient there was no difference and 1 patient had a $0.3-\mathrm{mm}$ worsening of herniation. Of the 2 patients with syrinxes, neither demonstrated resolution on 3-month postoperative imaging.

Preoperative T2-weighted imaging demonstrated restricted signal either anterior, posterior, or circumferentially around the cerebellar tonsils and spinal cord in 14 (77.8\%) of 18 patients. Cine flow imaging was obtained pre- and postoperatively for 8 patients (44.4\%) and only postoperatively for 10 patients $(55.6 \%)$. Eleven patients (61.1\%) demonstrated a postoperative improvement in CSF flow around the foramen magnum and/or cerebellar tonsils (Figs. 1 and 2). Four patients (22.2\%) did not show a change in CSF dynamics following treatment, but had adequate flow on both pre- and postoperative imaging. Three patients $(16.7 \%)$ continued to have restricted flow after decompression.

\section{Neurological Outcome and Radiographic Correlation}

We assessed patients' radiological changes relative to their clinical improvement and change in functional grade. There was no statistically significant difference in the relative change in posterior fossa area between patients with no, partial, or complete improvement in their symptoms (p $>0.05$ ). Patients with complete resolution of their symptoms had a significantly greater relative increase in their cisterna magna area following surgery than those with partial symptomatic improvement $(\mathrm{p}=0.022)$ (Fig. 3). Patients with a complete resolution of their symptoms had significantly smaller cisterna magna areas preoperatively compared with those who had no $(\mathrm{p}=0.025)$ or partial ( $\mathrm{p}$ $=0.020$ ) improvement (Fig. 4). The preoperative posterior fossa area was not significantly different between any of the groups.

Ten $(91 \%)$ of the 11 patients with improved CSF dynamics also had either a partial or complete resolution of their symptoms. All 4 of the patients with adequate but unchanged CSF flow experienced at least a partial improvement in their symptoms. Two (66.7\%) of the 3 patients who continued to have restricted flow postoperatively experienced a partial improvement, and the other had no change in clinical symptoms.
There were no significant effects of the degree of improvement in functional grade on radiological changes. One patient experienced a change of 3 grades (from Grade IV to Grade I); because she was the only patient to experience this substantial an improvement, she was excluded from the between-group comparisons.

\section{Surgical Complications}

There were no cases of CSF leak or postoperative infection (including meningitis). One patient experienced postoperative dysphagia and hypophonia, which were thought to be due to traumatic injury to her vocal cords during intubation. None of the patients, including those without clear symptom improvement, have since sought reoperation.

\section{Discussion}

Chiari malformation Type I is a common neurological ailment that often presents with headache and signs of brainstem compression, which is thought to be secondary to impairment in dynamic CSF flow. Patients with CM-I demonstrate a thickened dural band at the craniovertebral junction, with histological evidence of hyalinosis, calcification, and/or ossification. ${ }^{18}$ To restore CSF dynamics, surgical decompression is sought, with the mainstay of therapy being a posterior intradural decompression. In this case series, we demonstrate that an extradural decompression may be appropriate for increasing the important CSF spaces such that there is an improvement in CSF dynamics and consequently neurological symptoms.

\section{Extradural Chiari Decompression is a Viable and Reproducibly Effective Procedure}

Extradural decompression, while the most minimally invasive of the types of surgical decompression, nonetheless has been shown to be an effective technique for achieving symptom relief. ${ }^{5,9,11}$ Theoretically, the effectiveness of the dura-splitting technique depends on relieving the outer dural layer compression of the cord, and the expandability of the newly decompressed inner dural layer. Assessing the biomechanical properties of entire versus split dura mater, the former appears to be more fragile under stress, while the latter allows a greater volume of deformation at a lower stress level. ${ }^{6}$ In the case series by Isu et al., all 7 patients demonstrated $\mathrm{CM}-\mathrm{I}^{13}$ and then underwent a suboccipital craniectomy and either a $\mathrm{C} 1-2$ or C-1 laminectomy, followed by removal of the outer dural layer. The authors used intraoperative ultrasonography to confirm satisfactory decompression, as visualized by the pulsation of the cerebellar tonsils after removal of the outer dural layer. Six (86\%) of the 7 patients experienced improvement in their neurological symptoms, and within several months all 7 patients had a reduction in their syrinx size. This is congruent with our series, in which $88.9 \%$ of the patients experienced either partial or complete improvement in their symptoms. Chauvet et al. reported on a series of 11 patients treated surgically with extradural decompression, ${ }^{7}$ who initially presented with various symptoms, including headache and/or neck pain, secondary to brainstem compression and/or syringomyelia. All 11 pa- 


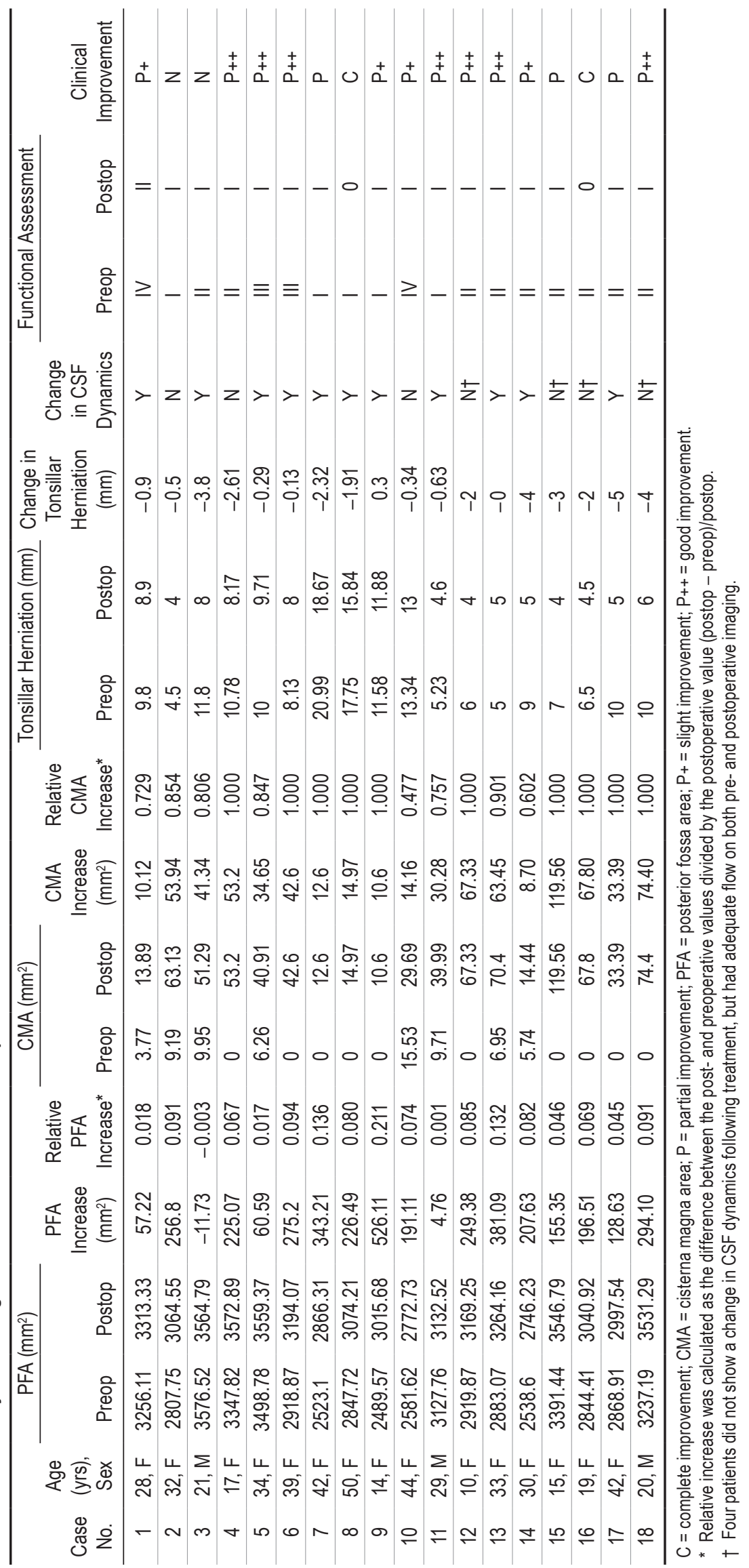



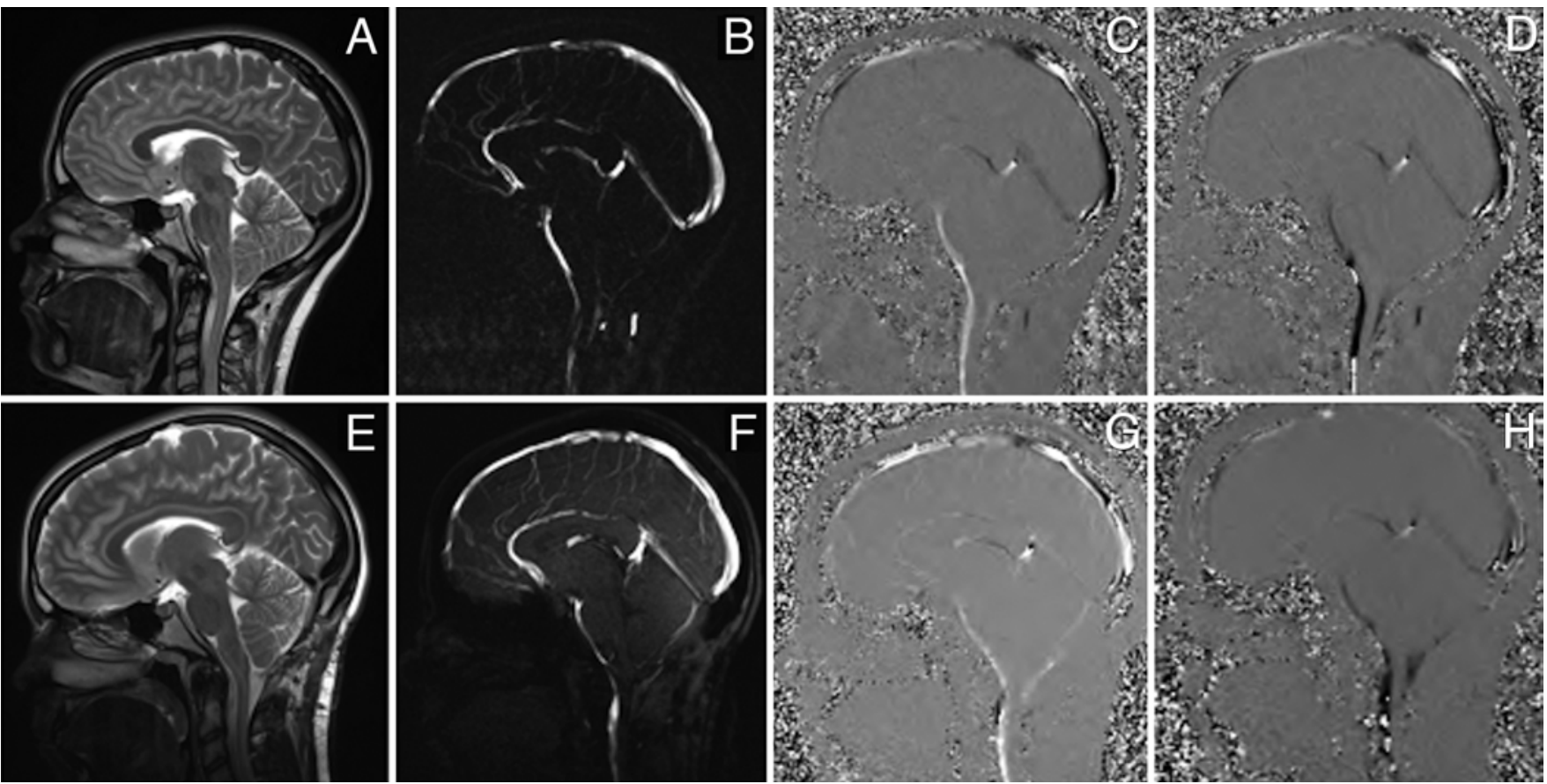

FIG. 1. Preoperative (A-D) and postoperative (E-H) sagittal MR images obtained in a representative patient with clinical and radiological improvement; T2-weighted ( $A$ and $E$ ), composite cine ( $B$ and $F$ ), phase-in cine (systole) $(C$ and $G$ ), and phase-out cine (diastole) ( $\mathrm{D}$ and $\mathrm{H})$. Postoperative T2-weighted imaging $(\mathrm{E})$ demonstrates some improvement in tonsillar herniation. Postoperative composite $(F)$, phase-in $(G)$, and phase-out $(H)$ cine demonstrate robust flow at the cervicomedullary junction and around the tonsils as well as improved biphasic flow. Flow in the cerebral aqueduct is minimally improved, and a blush of flow is now noted in the fourth ventricle.

tients experienced some improvement in symptoms, with $6(54.5 \%)$ having complete resolution. There were no fluid leaks or collections in the postoperative site and no cases of aseptic meningitis. Postoperative MRI showed that $10(91 \%)$ of the 11 patients had extensive decompression, with reestablishment of the cisterna magna in 4 patients, which is consistent with our findings. In our series, all 18 patients had an increase in cisterna magna area following extradural decompression. Furthermore, we found that patients with a complete resolution of their symptoms had a significantly greater increase in relative cisterna magna area compared with those with partial resolution of their symptoms. This difference did not hold true for the absolute increase in cisterna magna area. In fact, patients with complete symptom relief had a significantly smaller preoperative cisterna magna area compared with those patients with either partial or no symptom improvement. Our results suggest that if symptom relief is desired, adequate restoration of CSF spaces should be the goal of satisfactory decompression.

\section{Extradural Decompression is Safer and More Cost Effective}

When treating patients with CM-I without syringomyelia, some authors recommend posterior fossa decompression with external (partial-thickness) durotomy, and proceeding with intradural exploration only when adequate CSF flow cannot be achieved. ${ }^{8}$ In extradural decompression, some suggest using intraoperative ultrasonography to determine adequate CSF flow, ${ }^{8}$ whereas with intradural techniques it is difficult to assess CSF flow intraop- eratively. ${ }^{13}$ While there is no consensus on which surgical technique is more effective, ${ }^{5,9,15,20}$ an external decompression via splitting of the dura avoids the complications of a dura-penetrating craniectomy and laminectomy, ${ }^{7,13}$ where there is the potential for CSF leak and pseudomeningocele formation as well as meningitis..$^{13,15}$ The need for potential reoperation given inadequate symptom resolution is balanced by the lower complication rate. ${ }^{9}{ }^{14}$ However, others argue that the complication rate using an intradural opening with duraplasty is near negligible. ${ }^{20}$ In a comparison of duraplasty with intradural manipulation and dural splitting, the latter led to faster operating times, shorter hospital stays, and therefore lower total hospital costs while yielding comparable clinical results. ${ }^{15,16}$ More specifically, dural splitting in patients with CM-I without syringomyelia was deemed to be safer and more cost effective, not including the cost savings from the reduction in overall complications. ${ }^{16}$

\section{Preoperative Assessment and Prediction of Surgical Success-a Multimodal Approach}

Various techniques to assess the severity of compression, tonsillar herniation, and flow obstruction have been described to both predict and determine operative success. ${ }^{1,10}$ In their series of 11 patients undergoing intradural decompression without duraplasty, Noudel et al. measured the increase in posterior fossa volume (PFV) and correlated it with clinical outcomes. ${ }^{19}$ They found that the degree of PFV increase positively correlated with symptomatic improvement $(\mathrm{p}=0.014)$. There was a greater in- 

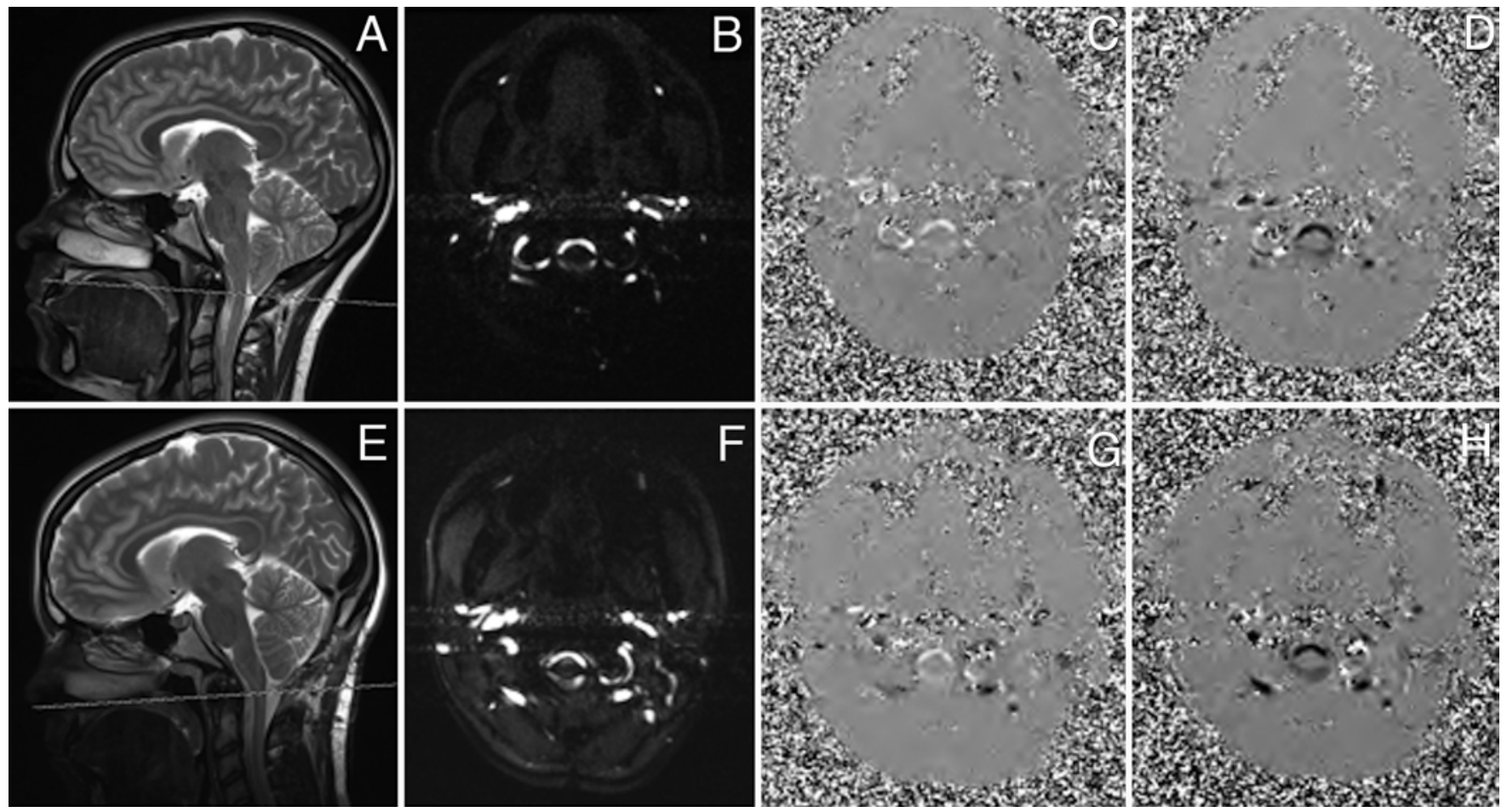

FIG. 2. Preoperative (A) and postoperative (E) T2-weighted sagittal MR images indicating the level of preoperative (B-D) and postoperative $(\mathrm{F}-\mathrm{H})$ axial cine flow MR images in a representative patient with clinical and radiological improvement. Composite cine ( $B$ and $F)$, phase-in cine (systole) ( $C$ and $G)$, and phase-out cine (diastole) (D and $H)$.

crease in PFV in patients with a smaller starting volume, and an increase of at least $15 \%$ was needed for a complete recovery. The authors further suggest that an optimal PFV increase could be predicted to improve patient symptoms. We did not find a correlation between posterior fossa area and symptom responsiveness, although this may be related to our dura-splitting technique and the fact that both layers of the dura were not opened in our series.

While traditional imaging techniques may be employed to assess the degree of tonsillar herniation and thus identify CM-I, they may not be sufficient when evaluating

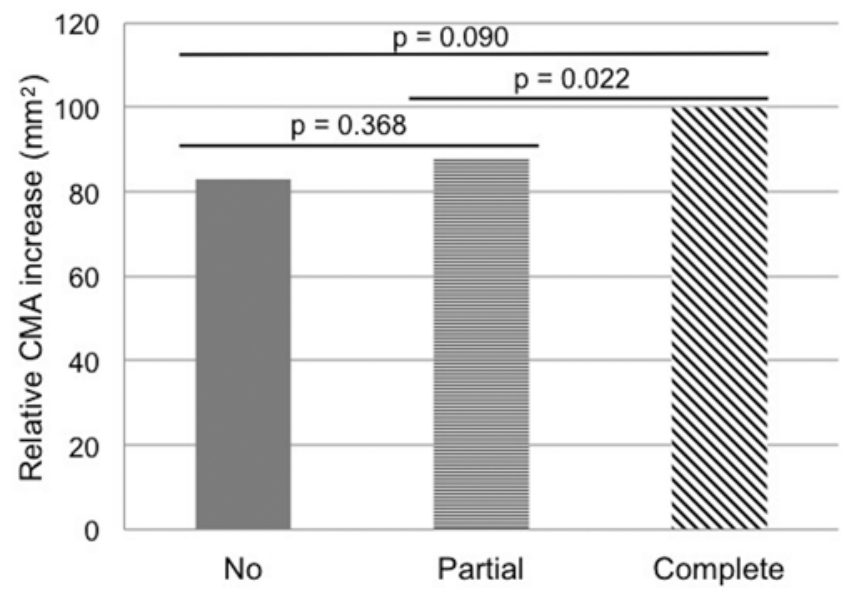

FIG. 3. Relative increase in cisterna magna area (CMA) across clinical improvement levels (no improvement, partial improvement, and complete improvement). whether to operate. ${ }^{14,21}$ After all, a "ball-valve" mechanism exacerbated by Valsalva-like maneuvers may be responsible for a dynamic obstruction of CSF flow and subsequent headache symptoms. ${ }^{14,21}$ Dynamic imaging studies therefore provide additional utility in assessing the degree of impairment. Some authors describe using ultrasonography with color Doppler imaging in addition to MRI in order to demonstrate anatomical compression as well as impaction of CSF flow. ${ }^{20}$

Phase-contrast cine MRI is an alternative study with even more clinical utility, given that a flow-sensitive pulse demonstrates the amplitude and direction of CSF and

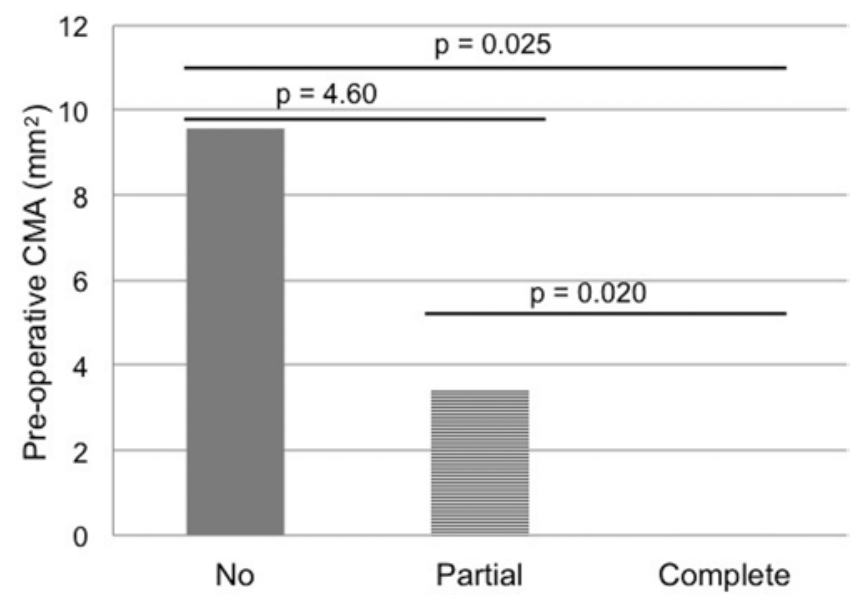

FIG. 4. Preoperative cisterna magna area across clinical improvement levels. 
blood flow relative to anatomical structures. ${ }^{21}$ Cine flow imaging may have the greatest utility in an asymptomatic or minimally symptomatic patient with an incidental finding of tonsillar herniation, when surgical treatment based only on abnormal anatomy has uncertain benefit. ${ }^{21}$ While traditional MRI is unable to capture Valsalva-related changes in CSF flow, cine flow can measure the more dynamic fluctuations relevant to patients with CSF obstruction. ${ }^{4}$ Although we were only able to obtain preoperative cine flow images for 8 (44.4\%) of 18 patients in this study, multiple studies have demonstrated that cine flow MRI has additional value for evaluating which patients are most likely to benefit from surgical intervention. Patients with Chiari malformation demonstrate abnormal CSF flow dynamics on cine compared with healthy controls, ${ }^{12}$ and cine parameters can be used to identify those with the disease. ${ }^{3}$ Ventureyra et al. showed that symptomatology correlated well with abnormal cine flow imaging. ${ }^{22}$ Asymptomatic patients almost invariably had normal findings on cine flow studies and did best without surgical treatment. Conversely, symptomatic patients treated with suboccipital decompression with duraplasty had improvement of symptoms, as well as improvement on postoperative imaging. ${ }^{22}$ Patients with abnormal CSF flow on preoperative cine are the most likely to have maximal benefit from surgical treatment, as these patients are less likely to have symptom recurrence. ${ }^{17}$ Of the 14 patients who had restricted flow preoperatively in our series, $12(85.7 \%)$ had either a partial or complete improvement in their symptoms. Nevertheless, of the 11 patients with improved flow, 10 reported at least a partial improvement in their symptoms. This suggests that restoration of CSF dynamics may be nonetheless beneficial. One limitation of cine flow MRI is that it only measures dependent flow, as the patient is lying down during the study. It is therefore possible that some patients may falsely show adequate flow that would otherwise be compromised in the standing position.

\section{Conclusions}

Extradural decompression provides the benefits of surgical decompression while avoiding the complications of intradural techniques. The dura-splitting surgical technique has the potential to be the first-line treatment for CM-I. Our data suggest that surgical enlargement of CSF spaces along with either adequate or improved CSF dynamics allows for the greatest chance of success following extradural decompression. Patients with either absent or severely reduced cisterna magna areas in addition to restricted flow might be the most likely to benefit from a basic decompression. In cases where patients remain symptomatic despite the lack of radiological findings, a more invasive technique might better address intradural pathology. Nevertheless, further studies with larger cohorts, ideally randomized to the 2 techniques (intradural vs extradural) would corroborate this study and hopefully bring the extradural Chiari decompression technique into the mainstream spotlight.

\section{References}

1. Arora P, Pradhan PK, Behari S, Banerji D, Das BK, Chhabra
DK, et al: Chiari I malformation related syringomyelia: radionuclide cisternography as a predictor of outcome. Acta Neurochir (Wien) 146:119-130, 2004

2. Baisden J: Controversies in Chiari I malformations. Surg Neurol Int 3 (Suppl 3):S232-S237, 2012

3. Bhadelia RA, Frederick E, Patz S, Dubey P, Erbay SH, DoDai D, et al: Cough-associated headache in patients with Chiari I malformation: CSF flow analysis by means of cine phase-contrast MR imaging. AJNR Am J Neuroradiol 32:739-742, 2011

4. Bhadelia RA, Madan N, Zhao Y, Wagshul ME, Heilman C, Butler JP, et al: Physiology-based MR imaging assessment of CSF flow at the foramen magnum with a valsalva maneuver. AJNR Am J Neuroradiol 34:1857-1862, 2013

5. Caldarelli M, Novegno F, Massimi L, Romani R, Tamburrini G, Di Rocco C: The role of limited posterior fossa craniectomy in the surgical treatment of Chiai malformation Type I: experience with a pediatric series. J Neurosurg (3 Suppl) 106:187-195, 2007

6. Chauvet D, Carpentier A, Allain JM, Polivka M, Crépin J, George B: Histological and biomechanical study of dura mater applied to the technique of dura splitting decompression in Chiari type I malformation. Neurosurg Rev 33:287-295, 2010

7. Chauvet D, Carpentier A, George B: Dura splitting decompression in Chiari type 1 malformation: clinical experience and radiological findings. Neurosurg Rev 32:465-470, 2009

8. Dlouhy BJ, Menezes AH: Osteopetrosis with Chiari I malformation: presentation and surgical management. J Neurosurg Pediatr 7:369-374, 2011

9. Durham SR, Fjeld-Olenec K: Comparison of posterior fossa decompression with and without duraplasty for the surgical treatment of Chiari malformation Type I in pediatric patients: a meta-analysis. J Neurosurg Pediatr 2:42-49, 2008

10. Erdogan E, Cansever T, Secer HI, Temiz C, Sirin S, Kabatas $\mathrm{S}$, et al: The evaluation of surgical treatment options in the Chiari malformation Type I. Turk Neurosurg 20:303-313, 2010

11. Genitori L, Peretta P, Nurisso C, Macinante L, Mussa F: Chiari type I anomalies in children and adolescents: minimally invasive management in a series of 53 cases. Childs Nerv Syst 16:707-718, 2000

12. Hentschel S, Mardal KA, Løvgren AE, Linge S, Haughton V: Characterization of cyclic CSF flow in the foramen magnum and upper cervical spinal canal with MR flow imaging and computational fluid dynamics. AJNR Am J Neuroradiol 31:997-1002, 2010

13. Isu T, Sasaki H, Takamura H, Kobayashi N: Foramen magnum decompression with removal of the outer layer of the dura as treatment for syringomyelia occurring with Chiari I malformation. Neurosurgery 33:845-850, 1993

14. Koyanagi I, Houkin K: Pathogenesis of syringomyelia associated with Chiari type 1 malformation: review of evidences and proposal of a new hypothesis. Neurosurg Rev 33:271285,2010

15. Limonadi FM, Selden NR: Dura-splitting decompression of the craniocervical junction: reduced operative time, hospital stay, and cost with equivalent early outcome. J Neurosurg 101 (2 Suppl):184-188, 2004

16. Litvack ZN, Lindsay RA, Selden NR: Dura splitting decompression for Chiari I malformation in pediatric patients: clinical outcomes, healthcare costs, and resource utilization. Neurosurgery 72:922-929, 2013

17. McGirt MJ, Atiba A, Attenello FJ, Wasserman BA, Datoo G, Gathinji M, et al: Correlation of hindbrain CSF flow and outcome after surgical decompression for Chiari I malformation. Childs Nerv Syst 24:833-840, 2008

18. Nakamura N, Iwasaki Y, Hida K, Abe H, Fujioka Y, Nagashima K: Dural band pathology in syringomyelia with Chiari 
type I malformation. Neuropathology 20:38-43, 2000

19. Noudel R, Gomis P, Sotoares G, Bazin A, Pierot L, Pruvo JP, et al: Posterior fossa volume increase after surgery for Chiari malformation Type I: a quantitative assessment using magnetic resonance imaging and correlations with the treatment response. J Neurosurg 115:647-658, 2011

20. Oró JJ, Mueller DM: Posterior fossa decompression and reconstruction in adolescents and adults with the Chiari I malformation. Neurol Res 33:261-271, 2011

21. Ramón C, Gonzáles-Mandly A, Pascual J: What differences exist in the appropriate treatment of congenital versus acquired adult Chiari type I malformation? Curr Pain Headache Rep 15:157-163, 2011

22. Ventureyra EC, Aziz HA, Vassilyadi M: The role of cine flow MRI in children with Chiari I malformation. Childs Nerv Syst 19:109-113, 2003

23. Whitney N, Sun H, Pollock JM, Ross DA: The human fora- men magnum-normal anatomy of the cisterna magna in adults. Neuroradiology 55:1333-1339, 2013

\section{Author Contributions}

Conception and design: all authors. Acquisition of data: DiLuna, Quon. Analysis and interpretation of data: Quon, Grant. Drafting the article: Quon. Critically revising the article: all authors. Reviewed submitted version of manuscript: all authors. Study supervision: DiLuna.

\section{Correspondence}

Michael L. DiLuna, Department of Neurosurgery, TMP4, Yale University School of Medicine, 333 Cedar St., New Haven, CT 06510.email: michael.diluna@yale.edu. 